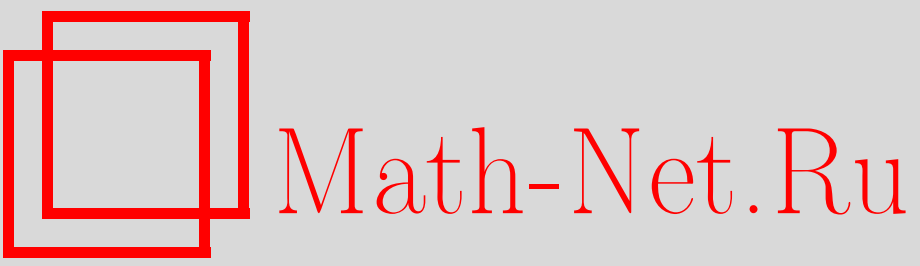

А. М. Боярский, Т. В. Скрыпник, Сингулярные орбиты присоединенного представления групп Ли $\mathrm{SO}(n)$, УMH, 1996, том 51, выпуск 3, 181-182

DOI: https://doi.org/10.4213/rm974

Использование Общероссийского математического портала Math-Net.Ru подразумевает, что вы прочитали и согласны с пользовательским соглашением

http://www. mathnet.ru/rus/agreement

Параметры загрузки:

IP: 44.207 .124 .84

26 апреля 2023 г., 04:30:00 


\title{
СИНГУЛЯРНЫЕ ОРБИТЫ ПРИСОЕДИНЕННОГО ПРЕДСТАВЛЕНИЯ ГРУПП ЛИ $\mathrm{SO}(n)$
}

\author{
А. Боярский, Т. Скрыпник
}

Рассмотрим алгебру Ли so $(n)$, реализованную как алгебра антисимметричных матриц со следом, равным нулю:

$$
\operatorname{so}(n) \ni X=\left(\begin{array}{cccc}
0 & x_{1} & \ldots & x_{n-1} \\
-x_{1} & 0 & \ldots & x_{2 n-2} \\
\ldots \ldots & \ldots & \ldots & \ldots \\
-x_{n-1} & \ldots & -x_{n(n-1) / 2} & 0
\end{array}\right)
$$

Как известно, орбиты присоединенного представления группы $\mathrm{SO}(n)$ задаются в $\mathbb{R}^{N}(N=$ $n(n-1) / 2)$ как совместные поверхности уровня системы функций Казимира

$$
S_{k}=\operatorname{Tr} X^{k}=\sum_{i_{1} \ldots i_{k}} X_{i_{1} i_{2}} X_{i_{2} i_{3}} \ldots X_{i_{k} i_{1}}, \quad k=\overline{1, l}, \quad l=[n / 2] .
$$

Иногда вместо этой системы удобно рассматривать эквивалентную систему функций, а именно коэффициенты $P_{k}$ характеристического многочлена

$$
\Delta_{X}(\lambda)=|X-\lambda E|=\lambda^{n}-P_{1} \lambda^{n-1}-P_{2} \lambda^{n-2}-\ldots-P_{n} .
$$

Переход от первой системы ко второй осуществляется при помощи формулы

$$
k P k=S_{k}-P_{1} S_{k-1}-\ldots-P_{k-1} S_{1}
$$

(см. [1]). Для $\operatorname{so}(n) S_{2 k+1}=\operatorname{Tr} X^{2 k+1}=0 \Rightarrow P_{2 k+1}=0$ и

$$
P_{2}=\frac{1}{2} S_{2}, \quad P_{4}=S_{4}-\frac{1}{2} S_{2}^{2}, \quad P_{6}=S_{6}-\frac{1}{4} S_{2} S_{4}+\frac{1}{8} S_{2}^{2}, \ldots .
$$

Лемма 1. Для $X \in \mathrm{so}(n)$ имеет место формула

$$
P_{n-k}=(-1)^{k} \sum_{i_{1}<\cdots<i_{k}}\left|A_{i_{1} \ldots i_{k}}^{i_{1} \ldots i_{k}}\right|=(-1)^{k} \sum\left(P f^{i_{1} \ldots i_{k}}\right)^{2},
$$

где $A_{i_{1} \ldots i_{k}}^{i_{1} \ldots i_{k}}$ - главные дополнительные миноры, получаемые из $X$ вычеркиванием $i_{1}, \ldots, i_{k}$ строк и $i_{1}, \ldots, i_{k}$ столбцов, а $P f^{i_{1} \ldots i_{k}}-$ пфаффиань этих миноров:

$$
P f^{i_{1} \ldots i_{k}}=\sum_{\substack{l_{1}<l_{2}, \ldots, l_{n-k-1}<l_{n-k} \\ l_{1}<\cdots<l_{n-k-1}}}(-1)^{\sigma} a_{l_{1} l_{2}} \ldots a_{l_{n-k-1} l_{n-k}}, \quad \text { əдe } \quad A^{i_{1} \ldots i_{k}}=\left\|a_{l_{m}}\right\| .
$$

ДокАЗАТЕЛЬСТво. Эта формула легко получается, если для матрицы $X-\lambda E$ в выражении для определителя через сумму по перестановкам привести подобные слагаемые при произведениях диагональных элементов.

Tеорема 1. Система уравнений $P_{1}=c_{1}, \ldots, P_{n-k+1}=c_{n-k+1}, P f^{i_{1} \ldots i_{k}}=0$ описывает явное вложение синглярных орбит присоединенного представления групп $\mathrm{SO}(n)$ следующих размерностей $m_{k}$ :

1) $n=2 l: m_{k}=2(l(l-1)-k(k-1)), k=\overline{1, l}$;

2) $n=2 l+1: m_{k}=2 l^{2}-2 k^{2}, k=\overline{1, l}$. 
ДокаЗАтельство. Ясно, что равенство $P_{n-k}=0$ эквивалентно системе $P f^{i_{1} \ldots i_{k}}=0$, $i_{1}=\overline{1, n}, \ldots, i_{k}=\overline{1, n}$. Поэтому можно предположить, что последняя система вместе с условиями постоянства остальных функций Казимира будет описывать орбиты меньшей размерности (так как увеличилось количество независимых функций).

Так как для so $(n)$ все орбиты проходят через подалгебру Картана $H$, для нахождения сингулярных орбит достаточно найти элементы этой подалгебры, имеющие аннуляторы большей, чем в случае общего положения, размерности (при этом одна орбита может пересекать подалгебру Картана в нескольких точках). Используя явный вид коммутационных соотношений и реализацию подалгебры Картана в виде антисимметричных антидиагональных матриц, можно увидеть, что орбиты размерности $m_{k}$ проходят через точки с $\alpha_{i_{1}}=\cdots=\alpha_{i_{k}}=0$, где $\alpha_{i}$ - антидиагональные матричные элементы матрицы из подалгебры $H$. Это орбиты вида

$$
\mathrm{SO}(2 n) /\left(\mathrm{SO}(2 k) * \mathrm{SO}(2)^{n-k}\right), \quad \mathrm{SO}(2 n+1) /\left(\mathrm{SO}(2 k+1) * \mathrm{SO}(2)^{n-k}\right)
$$

(здесь $\left(\mathrm{SO}(2)^{n-k}\right.$ обозначает $\mathrm{SO}(2)$ в степени $\left.n-k\right)$. Возвращаясь к уравнениям $P f^{i_{1} \ldots i_{k}}=0$, $i_{1}=\overline{1, n}, \ldots, i_{k}=\overline{1, n}$, заметим, что для пфафффианов имеется формула разложения по строке и столбцу, аналогичная формуле для определителей, и поэтому из равенства нулю всех пфафффианов степени $k$ следует равенство нулю пфаффианов старших степеней, следовательно, можно рассматривать эти системы уравнений по отдельности. Их решениями являются как раз описанные выше элементы $H$, через которые проходят вырожденные орбиты размерностей $m_{k}$. Таким образом, можно проверить, что не все уравнения из каждой системы независимы, однако системы имеют постоянный ранг и вместе с условиями постоянства остальных функций Казимира задают вложения в $\mathbb{R}^{n(n-1) / 2}$ указанных выше орбит.

Интересно отметить, что орбиты минимальной размерности во всех $\operatorname{so}(n)$ задаются квадратичными функциями. Например, для so $(5)$ уравнения $P f^{i}=0, i=\overline{1,5}$, имеют вид $(\vec{a} \vec{b})=0$; $(\vec{b} \vec{c})=0 ;[\vec{a} \times \vec{c}]+\vec{b} d=0$, где $\vec{a}, \vec{b}, \vec{c}, d$ - три трехмерных вектора и число, параметризующие 10 -мерную so(5). Легко видеть, что два скалярных уравнения почти везде следуют из векторного, и вместе с уравнением $\operatorname{Tr} X^{2}=a^{2}+b^{2}+c^{2}+d^{2}=$ const система определяет однопараметрическое семейство 6-мерных вырожденных орбит. Выбрать $\vec{a}, \vec{b}, \vec{c}, d$, не меняя вида уравнений, можно тремя способами. Таким образом, орбиту можно единообразно описывать в различных картах. Подробный анализ для so(5), so(6), so(7), а также обобщение этой конструкции на другие компактные алгебры изложены в [3].

\section{СПИСОК ЛИТЕРАТУРЫ}

[1] Гантмахер Ф. Р. Теория матриц. М.: Наука, 1967. [2] Гуревич Г. Б. Основы теории алгебраических инвариантов. М.: ОГИЗ, 1948. [3] Боярский А., Скрыпник Т. // Укр. матем. журн. 1996.

Принято редколлегией 11.03.1996 